\title{
Shox 2 influences mesenchymal stem cell fate in a co-culture model in vitro
}

\author{
YUANYUAN FENG ${ }^{1,2^{*}}$, PAN YANG ${ }^{1 *}$, SHOUMING LUO $^{1}$, \\ ZHIHUI ZHANG ${ }^{1}$, HUAKANG LI ${ }^{1}$, PING ZHU ${ }^{1}$ and ZHIYUAN SONG ${ }^{1}$ \\ ${ }^{1}$ Department of Cardiology, Southwest Hospital, Third Military Medical University, Chongqing 400038; \\ ${ }^{2}$ Department of Cardiology, The 401 Hospital of PLA, Qingdao, Shandong 266071, P.R. China
}

Received April 20, 2015; Accepted February 24, 2016

DOI: $10.3892 / \mathrm{mmr} .2016 .5306$

\begin{abstract}
Sinoatrial node (SAN) dysfunction is a common cardiovascular problem, and the development of a cell sourced biological pacemaker has been the focus of cardiac electrophysiology research. The aim of biological pacemaker therapy is to produce SAN-like cells, which exhibit spontaneous activity characteristic of the SAN. Short stature homeobox 2 (Shox2) is an early cardiac transcription factor and is crucial in the formation and differentiation of the sinoatrial node (SAN). The present study aimed to improve pacemaker function by overexpression of Shox 2 in canine mesenchymal stem cells (cMSCs) to induce a phenotype similar to native pacemaker cells. To achieve this objective, the cMSCs were transfected with lentiviral pLentis-mShox2-red fluorescent protein, and then co-cultured with rat neonatal cardiomyocytes (RNCMs) in vitro for 5-7 days. The feasibility of regulating the differentiation of cMSCs into pacemaker-like cells by Shox 2 overexpression was investigated. Reverse transcriptionquantitative polymerase chain reaction and western blotting showed that Shox2-transfected cMSCs expressed high levels of $\mathrm{T}$ box 3 , hyperpolarization-activated cyclic nucleotide-gated cation channel and Connexin 45 genes, which participate in SAN development, and low levels of working myocardium genes, Nkx2.5 and Connexin 43. In addition, Shox2-transfected cMSCs were able to pace RNCMs with a rate faster than the control cells. In conclusion, these data indicate that overexpression of Shox 2 in cMSCs can greatly enhance the pacemaker phenotype in a co-culture model in vitro.
\end{abstract}

Correspondence to: Professor Zhiyuan Song, Department of Cardiology, Southwest Hospital, Third Military Medical University, 30 Gaotanyan Road, Shapingba, Chongqing 400038, P.R. China E-mail: zysong2010@126.com

${ }^{*}$ Contributed equally

Key words: Shox2, neonatal cardiomyocytes, co-culture, mesenchymal stem cells, cardiomyogenic differentiation

\section{Introduction}

The primary treatment of sinoatrial node (SAN) dysfunction due to congenital defects, acquired diseases, gene mutations and aging is electronic pacemaker implantation. However, such devices are not optimal choices due to deficiencies in biological responsiveness and other shortages, such as lack of autonomic modulation, limited battery life, the need for permanent catheter implantation into the heart, unstable electrode position, and electronic and magnetic interference (1). A cell therapy approach to produce a biological pacemaker focuses on the overexpression of hyperpolarization-activated cyclic nucleotide-gated cation channel (HCN4) which can induce 'funny' current $I_{f}$. The $I_{f}$ current is known to be essential for the spontaneous diastolic depolarization of SAN cells (2-4). In our previous study, it was demonstrated that HCN4-transfected cMSCs in vivo can induce spontaneous activity; however, the spontaneous rates were lower than that of normal SAN cells (3). In addition, it was indicated that HCN4 loss was a cause of the dysfunction of SAN cells and resulted in abnormally slow heart rates (3-5). To overcome this obstacle, identification of an upstream gene is required in order to maintain the high expression of HCN4.

SAN development is a strictly regulated process, and a number of signaling molecules are involved. Shox2, a member of the short stature homeobox family, is an early cardiac transcription factor which has been identified to be uniquely expressed in the SAN region (6). Studies in Shox 2 knockout mouse models demonstrated that Shox 2 is crucial in the formation and differentiation of SAN by regulating the genetic cascade. A Shox 2 null mutation may lead to heart defects, including cardiac edema and hypoplasia of the SAN due to a reduced level of cell proliferation, which results in a decrease in the heart rate (6). Shox 2 mutation has also been shown to impact the SAN genetic network resulting in the downregulation of HCN4 in this region (6). Conversely, overexpression of Shox 2 in mice and Xenopus embryos showed a decreased expression of working myocardium markers (6,7). However, whether overexpression of Shox 2 in MSCs is able to establish a phenotype similar to native pacemaker cells and improve pacemaker function remains unknown.

The aim of the present study was to identify an upstream gene to maintain high expression of HCN4. A previous study 
demonstrated that Shox 2 is important in the differentiation of SAN and is an upstream gene of HCN4 (6). A number of studies have indicated that co-culture of MSCs with neonatal cardiomyocytes (CMs) can induce MSC differentiation into $\mathrm{CMs}$ in vitro, providing a model for heart tissue engineering research (8-10). Based on these data, canine MSCs (cMSCs) effectively transfected with a lentiviral vector encoding a mouse Shox 2 (mShox2) gene was employed in this study, and a model of direct co-culture of cMSCs with rat neonatal CMs (RNCMs) was established. Then levels of functional markers characterizing mature SAN cells, such as T box 3 (Tbx3), HCN4 and Connexin 45 (Cx45) were evaluated. It was investigated whether the regulation of the differentiation of cMSCs into pacemaker-like cells through the overexpression of Shox2 is feasible.

\section{Materials and methods}

cMSCs culture and identification. cMSCs were isolated from the bone marrow of three adult dogs (two males and one female; Third Military Medical University, Chongqing, China), weighing 10-14 kg, as previously described (3). In brief, bone marrow aspiration from the femurs and tibias was performed on dogs anesthetized with $30 \mathrm{mg} / \mathrm{kg}$ intravenous sodium pentobarbital (Sigma-Aldrich, St. Louis, MO, USA). The cells obtained were grown in $\alpha$-mimium essential medium (Hyclone Laboratories, Inc., Logan, UT, USA), supplemented with $0.22 \%$ HEPES, $0.22 \% \mathrm{Na}_{2} \mathrm{CO}_{3}, 10 \%$ fetal calf serum (Gibco; Thermo Fisher Scientific, Inc., Waltham, MA, USA), $100 \mathrm{U} / \mathrm{ml}$ penicillin and $100 \mu \mathrm{g} / \mathrm{ml}$ streptomycin (Hyclone Laboratories, Inc.), and incubated in a $37^{\circ} \mathrm{C}, 5 \%$ $\mathrm{CO}_{2}$ humidified atmosphere. These cMSCs were identified via flow cytometry with $\mathrm{CD} 34^{-} / \mathrm{CD} 45^{-} / \mathrm{CD} 29^{+} / \mathrm{CD} 44^{+}$and for the ability to differentiate into adipogenic, osteogenic and chondrogenic lineages as previously described (3). The study was approved by the ethics committee of the Third Military Medical University.

Construction of mShox 2 lentiviral vector and mShox 2 infection. The lentiviral vector expressing $\mathrm{mShox} 2$ (pLentis-mShox2-RFP) was constructed by inserting the mShox 2 gene into a pLentis-RFP vector using BamHI (FD0054) and EcoRI (N41890) restriction sites, all obtained from Invitrogen (Thermo Fisher Scientific, Inc.). The lentiviral particles were prepared using a calcium phosphate method, as previously described (2-4). Third generation cMSCs were transfected with pLentis-mShox2-RFP or pLentis-RFP in the presence of $2 \mu \mathrm{g} / \mathrm{ml}$ polybrene (Sigma-Aldrich) at a multiplicity of infection (MOI) of 20 for $24 \mathrm{~h}$. The expression of RFP after $48 \mathrm{~h}$ was $>90 \%$ of the infected cells.

Co-culture conditions. Primary RNCMs were isolated from newborn Sprague Dawley rats in 2 days following by the steps used in our lab (11). Rats were obtained from the Third Military Medical University. Animal experiments were performed in accordance with the Guide for the Care and Use of Laboratory Animals published by the Third Military Medical University and approved by the Committee on the Ethics of Animal Experiments of the Third Military Medical University (permit number: SYXK2013-0012). Experiments were conducted at least in triplicate and $>30$ rats were used. Briefly, RNCMs were isolated by digestion with $1 \mathrm{mg} / \mathrm{ml}$ type I collagenase (Sigma-Aldrich) with $0.08 \%$ trypsin (Ameresco, Solon, $\mathrm{OH}$, USA) diluted with $\mathrm{Ca}^{+} \mathrm{Mg}^{+}$-free D-Hanks (Wuhan Boster Biological Technology, Ltd., Wuhan, China). The harvested RNCMs were purified by pre-plating $\left(30 \mathrm{~min}, 37^{\circ} \mathrm{C}\right)$. For co-culture experiments, cMSCs and RNCMs were mixed and plated at a ratio of 1:4 (20\% cMSCs) onto the $60-\mathrm{cm}^{2}$ culture dishes (Corning, Inc., Corning, NY, USA). The cell mixtures were co-cultured for 5-7 days as isotropic monolayers, with a medium change after $24 \mathrm{~h}$. Fluorescence mapping was performed after 5 days in culture, and cMSCs were identified with red fluorescence observed using a BX41 microscope (Olympus Corporation, Tokyo, Japan). RNCMs mature to form rod-shaped striated cells after $48 \mathrm{~h}$ in culture. The co-culture experiment was performed three times to validate the results. For reverse transcription-quantitative polymerase chain reaction (RT-qPCR) and western blotting, the cMSCs were selected by treatment with puromycin ( $3 \mu \mathrm{g} / \mathrm{ml}$; Sigma-Aldrich) for 3 days in addition to fluorescence-activated cell sorting for RFP.

Immunofluorescence. Immunofluorescence was conducted as previously described (11). Transfected cMSCs were fixed with 4\% paraformaldehyde (Wuhan Boster Biological Technology, Ltd., Wuhan, China) for $15 \mathrm{~min}$ at room temperature, washed in phosphate-buffered saline, then treated with $0.2 \%$ Triton (Sigma-Aldrich) for $15 \mathrm{~min}$. Cells were incubated with goat polyclonal anti-Shox2 (cat. no. sc-21898, Santa Cruz Biotechnology Inc., Santa Cruz Biotechnology Inc.; 1:50) and rabbit polyclonal anti-Cx45 (cat. no. sc-25716, Santa Cruz Biotechnology Inc.; 1:50) primary antibodies overnight at $4^{\circ} \mathrm{C}$. They were then incubated with donkey anti-goat IgG antibodies conjugated to Alexa Fluor 488 (cat. no. A-11055, Invitrogen, Thermo Fisher Scientific, Inc.; 1:100) for Shox2 and donkey anti-rabbit IgG antibody conjugated to Alexa Fluor 488 (A21206, Invitrogen, Thermo Fisher Scientific, Inc.; 1:100) for $\mathrm{Cx} 45$ for $60 \mathrm{~min}$. After further washing, the cells were mounted with antifade mounting medium (Beyotime Institute of Biotechnology, Shanghai, China). The nuclei stained with 4',6-diamidino-2-phenylindole (Sigma-Aldrich) was used as a location control. The fluorescent images were obtained with a Zeiss LSM710 laser confocal microscope (Carl Zeiss Microscopy GmbH, Jena, Germany). The results were analyzed with ZEN lite 2011 software (Carl Zeiss Microscopy $\mathrm{GmbH})$.

RT-qPCR analyses. RT-qPCR was performed according a previous study (12). All primers (Table I) were synthesized by Invitrogen, Thermo Fisher Scientific, Inc. (Shanghai, China). The total cellular mRNA was extracted with TRIzol (Invitrogen Thermo Fisher Scientific, Inc.). Subsequently, cDNA synthesis was performed according to the manufacturer's instructions. RT-qPCR was performed with SYBR Green Realtime PCR Master mix (QPK-201, Toyobo, Co., Ltd., Osaka, Japan) on a Stratagene Mx3000P (Agilent Technologies, Inc., Santa Clara, CA, USA) instrument. Quantitative measurements were determined using the comparative $\mathrm{Cq}\left(2^{-\Delta \Delta \mathrm{Cq}}\right)$ method (13). All samples were normalized by endogenous level of glyceraldehyde 3-phosphate dehydrogenase (GAPDH). All results were repeated three times. 
Table I. Primers used in reverse transcription-quantitative polymerase chain reaction analyses.

\begin{tabular}{lll}
\hline Gene & \multicolumn{1}{c}{ Forward } & \multicolumn{1}{c}{ Reverse } \\
\hline Shox2 & 5'-ACTATCCAGACGCTTTCATGCG-3' & 5'-TTCGATTTTGAAACCAAACCTGTAC-3' \\
Tbx3 & 5'-GTAAGATGTTCTGGGCTGGATAAA-3' & 5'-GTAGCAGGGCTGTCTGGG TG-3' \\
HCN4 & 5'-AGGGCACCATCGGCAAGA-3' & 5'-CCACGCTCAGCGAATACAGG-3' \\
Cx45 & 5'-CAGCAGACTTCCTTGCCCTCATA-3' & 5'-CTTAGCATTGGACAGTTCGGTGT-3' \\
Nkx2.5 & 5'-CCGAGCCTGGTAGGAAAGGG-3' & 5'-AAATCCAAGGGACGTGGAGACA-3' \\
Cx43 & 5'-TGCTATGACAAATCCTTCCCAATC-3' & 5'-GCCGTGCTCTTCAATTCCATACTT-3' \\
GAPDH & 5'-GAGATCCCGCCAACATCAAA-3' & 5'-GGCATCAGCAGAAGGAGCAG-3' \\
\hline
\end{tabular}

Shox2, Short stature homeobox 2; Tbx3, T box 3; HCN4, hyperpolarization-activated cyclic nucleotide-gated cation channel; Cx45, connexin 45; Cx43, Connexin 43; GAPDH, glyceraldehyde 3-phosphate dehydrogenase.

Western blotting. Western blotting was conducted as previously described (11). Briefly, cMSCs, washed in PBS, were lysed in radioimmunoprecipitation assay buffer containing phenylmethylsulfonyl fluoride (RIPA/PMSF), then the total protein in the sample was quantified using a bicinchoninic acid protein assay kit (Beyotime Institute of Biotechnology), according to the manufacturer's instructions. Briefly, the membranes were blocked with $5 \%$ bovine serum albumin (Gibco; Thermo Fisher Scientific, Inc.) in TBS solution for $3 \mathrm{~h}$ at room temperature with shaking. The membranes were then incubated with primary goat polyclonal antibody against Shox2 (cat. no. sc-21898, Santa Cruz Biotechnology Inc.; 1:200) and GAPDH (cat. no. sc-48166, Santa Cruz Biotechnology Inc.; 1:200) and rabbit polyclonal antibodies against Tbx3 (cat. no. sc-48781, Santa Cruz Biotechnology Inc.; 1:200), Cx45 (cat. no. sc-25716, Santa Cruz Biotechnology Inc.; 1:200), Cx43 (cat. no. sc-9059, Santa Cruz Biotechnology Inc.; 1:200), HCN4 (cat. no. ab69054, Abcam, Cambridge, UK; 1:100) and Nkx2.5 (cat. no. ab97355, Abcam, UK; 1:500) were incubated separately overnight at $4^{\circ} \mathrm{C}$ with gentle agitation. After $>3$ washes in Tris-buffered saline with Tween-20, the membranes were incubated for $2 \mathrm{~h}$ at room temperature with corresponding secondary antibodies: Donkey anti-goat IgG-horseradish peroxidase (HRP) (cat no. sc-2020, Santa Cruz Biotechnology Inc.; 1:5,000) and mouse anti-rabbit IgG-HRP (cat no. sc-2357; Santa Cruz Biotechnology Inc.; 1:5,000). Then specific bands of target proteins were visualized using an enhanced chemiluminescence detection kit (Beyotime Institute of Biotechnology) according to the manufacturer's recommendations. Finally, the target signals were normalized to the GAPDH signal. Experiments were performed several times to verify results.

Statistical analysis. Data are presented as the mean \pm standard error of the mean. Statistical comparisons among multiple groups were analyzed by one-way analysis of variance with Dunnett's T3 test with SPSS 19.0 software (IBM, Armonk, $\mathrm{NY}, \mathrm{USA}) . \mathrm{P} \leq 0.05$ was considered to indicate a statistically significant difference.

\section{Results}

Infection, expression, co-culture of cMSCs and morphological changes. At 2 days after transfection, the infected cMSCs exhibited red fluorescence. The transfection rates of cMSCs were $94 \pm 2.5 \%$, which was analyzed by confocal laser microscope images in at least four different random fields. The Shox2-RFP transfected cMSCs expressed Shox 2 protein (Fig. 1A), in concordance with the result obtained by Liu et al (14). The growth rate of these cMSCs decreased. In addition, their morphology was observed to change and some cells were long-rod or furcation shaped. After co-culture with RNCMs, the cMSCs were larger with spindle and spider-like morphologies.

Fluorescence microscopy revealed that cMSCs were randomly distributed in culture and that a large number existed in a plane below the RNCMs with some interspersed between the RNCMs (Fig. 1B). This is perhaps due to the fact that cMSCs adhere to the coverslips within 3 to $4 \mathrm{~h}$, whereas RNCMs take up to $24 \mathrm{~h}$ to fully adhere.

An important characteristic of cMSCs is their ability to assemble gap junctions between themselves and with neighboring CMs (15). Connexin molecule Cx45 is a marker of the SAN, Fig. 1C shows Cx45 expression in cMSC cultures by immunofluorescence. It was demonstrated that the expression of Cx45 in Shox2-transfected cMSCs co-cultured with RNCMs was higher than that of the control cells.

Co-culturing Shox2-transfected cMSCs with RNCMs upregulates $S A N$-marker expression. To investigate the role of Shox 2 in the differentiation of SAN, the expression status of several genes was investigated. These genes included those that have been used to identify SAN differentiation or are known to be important for SAN formation and function, including Tbx 3, a transcription factor expressed in SAN with a role in SAN development; HCN4, a molecular marker of pacemaker cells; and Cx45, which prevents the areas of conductivity inside the SAN. The results demonstrated that overexpression of Shox 2 significantly increased the expression of Tbx3, HCN4 and Cx45 at the mRNA $(\mathrm{P}<0.05)$ and protein levels, and the difference increased markedly when co-cultured with RNCMs (Figs. 2 and 3).

The effects of Shox 2 on the expression changes of two working myocardium markers $\mathrm{Nkx} 2.5$ and $\mathrm{Cx} 43$ in cMSCs co-cultured with RNCMs were also examined. As shown in Fig. 2, Nkx2.5 and Cx43 mRNA were downregulated in the Shox 2 overexpression cMSCs compared with the negative control group, and the difference was statistically significant in the co-culture group $(\mathrm{P}<0.05)$. By contrast, the expression of 
A

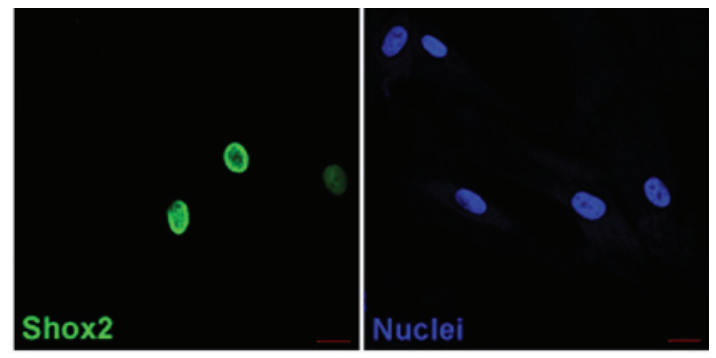

B

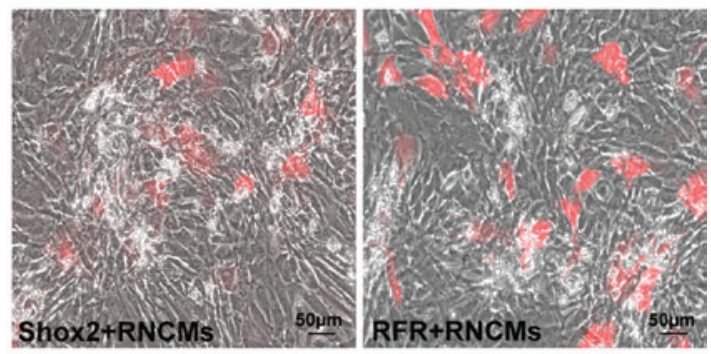

C
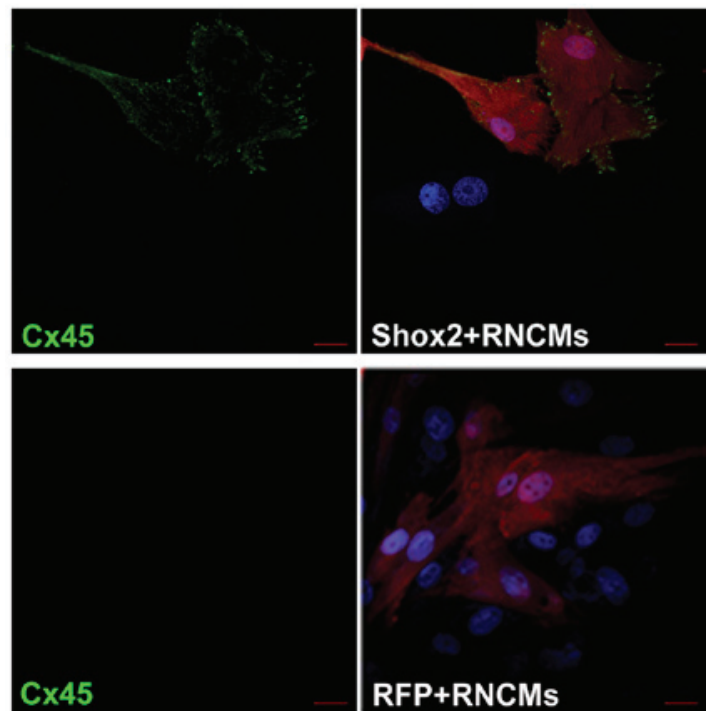

Figure 1.Characterization of cMSCs. (A) Laser confocal microscopy of cMSCs after transfection. cMSCs were transfected with pLentis-mShox2-RFP, as evidenced by the expression of Shox 2 . Nuclei were stained blue with 4',6-diamidino-2-phenylindole as a control. (B) Fluorescence images of 1:4 cMSCs: RNCMs co-cultured on day 5 after plating. cMSCs were randomly distributed in culture. Scale bar, $50 \mu \mathrm{m}$. (C) Shox2-transfected cMSCs expressing Cx45 were co-cultured with RNCMs. None of the control cells displayed this positive expression. Scale bar, $20 \mu \mathrm{m}$. cMSC, canine mesenchymal stem cells; NRMCs, rat neonatal cardiomyocytes; Shox2, shor stature homeobox 2; Cx45, Connexin 45; RFP, red fluorescent protein.

Nkx2.5 and Cx43 were significantly increased in RFP-cMSCs co-cultured with RNCMs at the mRNA and protein levels (Figs. 2 and 3, $\mathrm{P}<0.05$ ), in concordance with results obtained by Li et al (9).

Shox2-transfected cMSCs drive the rate of co-cultured $R N C M s$. It was then evaluated whether overexpression of Shox 2 in cMSCs can result in a pacemaker phenotype. After 5 days in co-culture, when a syncytium was established, the beat rates were counted. The mean rate was $(167 \pm 38 \mathrm{bpm}$, $\mathrm{n}=6$ ) in RNCMs co-cultured with Shox2-transfected cMSCs, which was markedly higher than the rate obtained from RNCMs co-cultured with control cells $(79 \pm 15 \mathrm{bpm}, \mathrm{n}=5)$ (Fig. 1B).

\section{Discussion}

In the present study, cMSCs overexpressing Shox 2 were co-cultured with RNCMs. The results demonstrated the functional role of Shox 2 in pacemaker cell differentiation, which indicated that overexpression of Shox 2 in cMSCs can greatly enhance the pacemaker phenotype in a co-culture model in vitro.

In our previous study, it was demonstrated that HCN4 transfected cMSCs can induce spontaneous activity in vivo; however, the spontaneous rates were lower than those in the normal SAN cells(3). It was hypothesized that this may be due to engrafted HCN4 gene loss in the host heart microenvironment (3-5). In our previous study, it was confirmed that overexpression of Shox 2 in cMSCs could upregulate $\mathrm{HCN} 4$ expression, and its level was significantly increased by co-culture induction. This was accompanied by altered expression of several other genes essential for SAN formation. Previous studies have demonstrated high expression levels of early transcription factor Tbx 3 and low levels of conductance gap junction protein $\mathrm{Cx} 45$ in the $\operatorname{SAN}(9,16,17)$. By contrast, the expression of transcription factor Nkx2.5 and high conductance gap junction protein Cx43 is widely observed in working myocardium but not in $\operatorname{SAN}(7,9)$. Increased Tbx3, HCN4 and Cx45 expression, and loss of $\mathrm{Nkx} 2.5$ and $\mathrm{Cx} 43$ expression indicated the formation of SAN-like cells $(6,7,16,17)$. In this study, it was indicated that $\mathrm{Tbx} 3$ and $\mathrm{Cx} 45$ were significantly upregulated in mShox 2-RFP transfected cMSCs. Additionally, co-culturing with RNCMs enhanced this effect and it was accompanied by the downregulated expression of $\mathrm{Nkx} 2.5$ and $\mathrm{Cx} 43$. Co-culturing with RNCMs was shown to provide a model mimicking the physiological microenvironment of the heart and constructed Shox2-cMSCs were able to differentiate into SAN like cells when co-cultured with RNCMs.

Genetically, SAN is a complicated and tightly regulated process including a variety of signaling molecules. Tbx 3 is a member of the T-box family, particularly expressed in the cardiac conduction system, including in the SAN, and is crucial during heart embryogenesis. Previous studies have suggested that $\mathrm{Tbx} 3$ can repress the expression of chamber-specific genes, such as $\mathrm{Cx} 40, \mathrm{Cx} 43$ and Nppa, and promote the pacemaker phenotype (16). By contrast, in Tbx3 mutants these markers may span the entire SAN and cause lethal arrhythmias: sinus pauses, bradycardia, atrioventricular block and sudden death (18). In this study, it was demonstrated that Tbx3 was significantly upregulated in mShox 2 transfected cMSCs and co-culturing with RNCMs enhanced this effect. In addition to the downregulation of Tbx 3 in heart tissue with Shox2 null mutation as reported by Espinoza-Lewis et al (6), it was hypothesized in the present study that Shox 2 may act earlier than Tbx 3 in pacemaker differentiation. In addition, $\mathrm{Cx} 45$ was also upregulated in Shox2-transfected cMSCs in this study, and the difference became greater when co-cultured with RNCMs. Cx45 is a member of the connexin gene family that can form gap junctions with low conductance preventing the suppressing hyperpolarizing influence of the atrium, and is seen as another marker of SAN $(19,20)$. Therefore, it was suggested that Shox 2 can promote pacemaker differentiation by enhancing the expression of Tbx 3 and $\mathrm{Cx} 45$ in vitro. 
Shox2

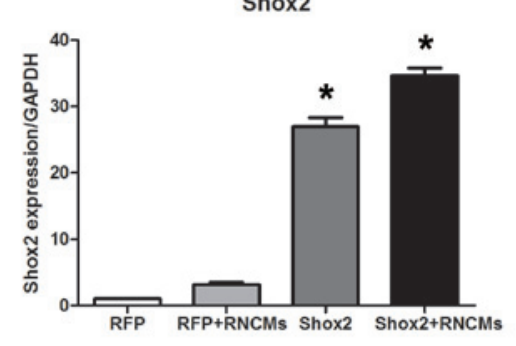

HCN4
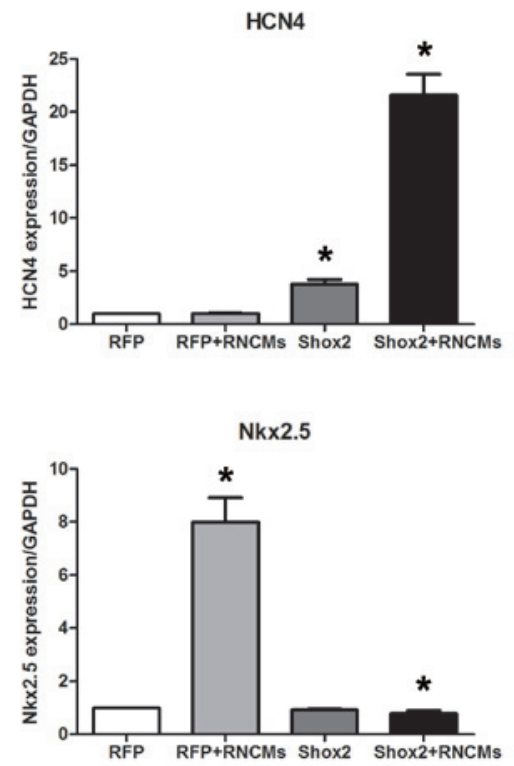

Tbx3

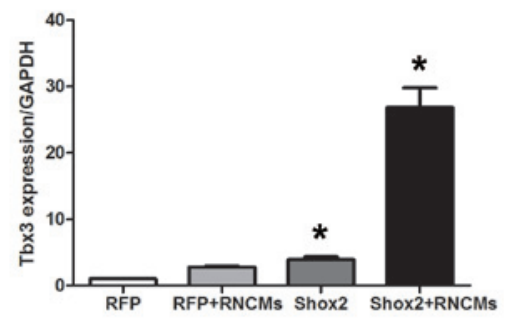

Cx45
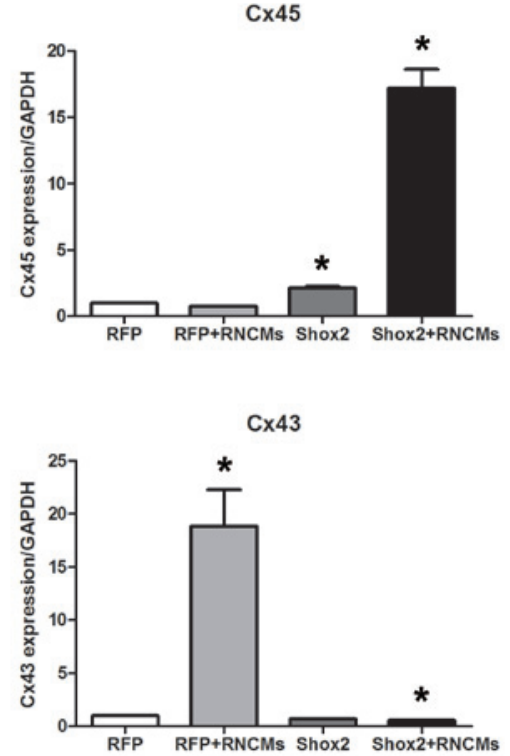

Figure 2. Shox2, Tbx3, HCN4, Cx45, Nkx2.5 and Cx43 gene expression was examined using reverse transcription-quantitative polymerase chain reaction. Similar results were obtained in three independent experiments. Data are presented as the mean \pm standard error of the mean. * $\mathrm{P}<0.05$ vs. control. Shox 2 , Short stature homeobox 2; Tbx3, T box 3; HCN4, hyperpolarization-activated cyclic nucleotide-gated cation channel; Cx45, connexin 45; Cx43, Connexin 43; RFP, red fluorescent protein; GAPDH, glyceraldehyde 3-phosphate dehydrogenase; RNCMs, rat neonatal cardiomyocytes.

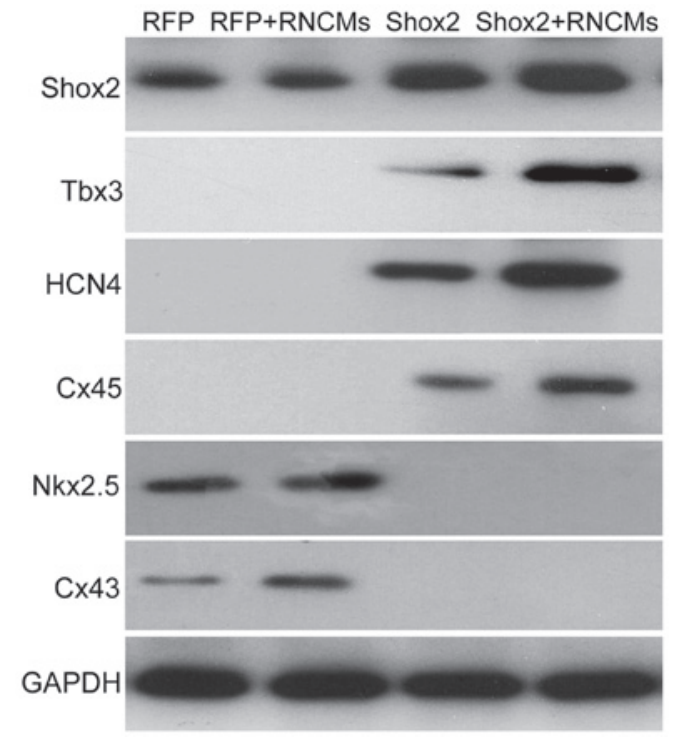

Figure 3. Shox2, Tbx3, HCN4, Cx45, Nkx2.5 and Cx43 protein expression were examined using western blotting. Similar results were obtained in three independent experiments. Shox2, Short stature homeobox 2; Tbx3, T box 3; HCN4, hyperpolarization-activated cyclic nucleotide-gated cation channel; Cx45, connexin 45; Cx43, Connexin 43; RFP, red fluorescent protein; GAPDH, glyceraldehyde 3-phosphate dehydrogenase; RNCMs, rat neonatal cardiomyocytes.
In this study, another cardiac transcription factor $\mathrm{Nkx} 2.5$ was also examined, which is critical for working myocardium differentiation but is not expressed in SAN $(7,9)$. Previous studies have shown that ectopic expression of $\mathrm{Nkx} 2.5$ can suppress the formation of SAN in the following ways: i) Histologically, hypoplastic SAN is observed to be attributed to reduced cell proliferation, thinned atrial wall and thickened ventricular wall; ii) functionally, overexpression of $\mathrm{Nkx} 2.5$ in the heart results in a reduced heartbeat rate; and iii) genetically, $\mathrm{Nkx} 2.5 \mathrm{can}$ induce differentiation of working myocardium, resulting in downregulation of $\mathrm{HCN} 4$ and Tbx 3 , and ectopic expression of $\mathrm{Cx} 40$ and Nppa in the SAN region (7). These changes in histology, function and genetics are consistent with the findings observed in Shox2-deficient embryonic hearts (6). Recently, it has been demonstrated that the expression patterns of Nkx2.5 and Shox 2 are mutually exclusive during SAN formation (14). Indeed, Shox 2 injected in mice and Xenopus embryos may lead to a downregulation of Nkx2.5 (6), and it was also indicated that overexpression of Shox 2 in cMSCs in vitro inhibited the expression of $\mathrm{Nkx} 2.5$ when co-culturing with RNCMs. Thus, Shox 2 may act as an $\mathrm{Nkx} 2.5$ repressor to regulate SAN differentiation. The effect of Shox2-overexpression on $\mathrm{Nkx} 2.5$ causes cells to differentiate into pacemaker-like cells rather than working myocardium. 
Taking Tbx3 and HCN4 as downstream of Nkx2.5, it may be possible to improve pacemaker function by affecting the levels of Shox2, Nkx2.5, Tbx3, HCN4, Cx43 and Cx45. In this network, Shox 2 firstly inhibits Nkx2.5 expression and activates the pacemaker differentiation program. Then, Tbx3, HCN4 and Cx45 are in turn expressed. In addition, the activation of Tbx 3 further inhibits the expression of $\mathrm{Cx} 43$.

In conclusion, the results indicated that overexpression of Shox 2 can regulate the differentiation of cMSCs into pacemaker-like cells and promote pacemaker function in vitro. This offers a good model for the development of biological pacemakers. Additionally, this study provides a basis for future in vivo experiments in dogs using Shox2-transfected cMSCs, and insight into future gene-targeted and regenerative therapeutic strategies for SAN dysfunction in humans.

\section{Acknowledgements}

This study was supported by the National Natural Science Foundation of China (grant no. 81270246).

\section{References}

1. Miake J, Marbán E and Nuss HB: Biological pacemaker created by gene transfer. Nature 19: 132-133, 2002.

2. Tong S, Yao Q, Wan Y, Zhou J, Shu M, Zhong L, Li Y, Zhang Q, Yindai J and Song Z: Development of functional I f channels in mMSCs after transfection with mHCN4: Effects on cell morphology and mechanical activity in vitro. Cardiology 112 114-121, 2009

3. Jun C, Zhihui Z, Lu W, Yaoming N, Lei W, Yao Q and Zhiyuan S: Canine bone marrow mesenchymal stromal cells with lentiviral mHCN4 gene transfer create cardiac pacemakers. Cytotherapy 14: 529-539, 2012.

4. Lu W, Yaoming N, Boli R, Jun C, Changhai Z, Yang Z and Zhiyuan S: mHCN4 genetically modified canine mesenchymal stem cells provide biological pacemaking function in complete dogs with atrioventricular block. Pacing Clin Electrophysiol 36: $1138-1149,2013$

5. Cai J, Yi FF, Li YH, Yang XC, Song J, Jiang XJ, Jiang H, Lin GS and Wang W: Adenoviral gene transfer of HCN4 creates a genetic pacemaker in pigs with complete atrioventricular block. Life Sci 80: 1746-1753, 2007.

6. Espinoza-Lewis RA, Yu L, He F, Liu H, Tang R, Shi J, Sun X, Martin JF, Wang D, Yang J and Chen Y: Shox 2 is essential for the differentiation of cardiac pacemaker cells by repressing Nkx2-5. Dev Biol 327: 376-385, 2009 .
7. Espinoza-Lewis RA, Liu H, Sun C, Chen C, Jiao K and Chen Y: Ectopic expression of $\mathrm{Nkx} 2.5$ suppresses the formation of the sinoatrial node in mice. Dev Biol 356: 359-369, 2011.

8. Chang MG, Tung L, Sekar RB, Chang CY, Cysyk J, Dong P, Marbán E and Abraham MR: Proarrhythmic potential of mesenchymal stem cell transplantation revealed in an in vitro coculture model. Circulation 113: 1832-1841, 2006.

9. Li Y, Li B, Zhang C, Zhang J, Zeng M and Zheng Z: Effect of NRG-1/ErbB signaling intervention on the differentiation of bone marrow stromal cells into sinus node-like cells. J Cardiovasc Pharmacol 63: 434-440, 2014.

10. Wang T, Xu Z, Jiang W and Ma A: Cell-to-cell contact induces mesenchymal stem cell to differentiate into cardiomyocyte and smooth muscle cell. Int J Cardiol 109: 74-81, 2006.

11. Wen L, Zhang C, Nong Y, Yao Q and Song Z: Mild electrical pulse current stimulation upregulates S100A4 and promotes cardiogenesis in MSC and cardiac myocytes coculture monolayer. Cell Biochem Biophys 65: 43-55, 2013.

12. Kim MO, Jung H, Kim SC, Park JK and Seo YK: Electromagnetic fields and nanomagnetic particles increase the osteogenic differentiation of human bone marrow-derived mesenchymal stem cells. Int J Mol Med 35: 153-160, 2015.

13. Livak KJ and Schmittgen TD: Analysis of relative gene expression data using real-timequantitative PCR and the 2(-Delta Delta C(T)) Method. Methods 25: 402-408, 2001.

14. Liu H, Chen $\mathrm{CH}$, Ye W, Espinoza-Lewis RA, Hu X, Zhang Y and Chen Y: Phosphorylation of Shox 2 is required for its function to control sinoatrial node formation. J Am Heart Assoc 3: e000796, 2014.

15. Valiunas V, Doronin S, Valiuniene L, Potapova I, Zuckerman J, Walcott B, Robinson RB, Rosen MR, Brink PR and Cohen IS: Human mesenchymal stem cells make cardiac connexins and form functional gap junctions. J Physiol 555: 617-626, 2004.

16. Hoogaars WM, Engel A, Brons JF, Verkerk AO, de Lange FJ, Wong LY, Bakker ML, Clout DE, Wakker V, Barnett P, et al: Tbx3 controls the sinoatrial node gene program and imposes pacemaker function on the atria. Genes Dev 21: 1098-1112, 2007.

17. Wiese C, Grieskamp T, Airik R, Mommersteeg MT, Gardiwal A, de Gier-de Vries C, Schuster-Gossler K, Moorman AF, Kispert A and Christoffels VM: Formation of the sinus node head and differentiation of sinus node myocardium are independently regulated by Tbx18 and Tbx3. Circ Res 104: 388-397, 2009.

18. Frank DU, Carter KL, Thomas KR, Burr RM, Bakker ML, Coetzee WA, Tristani-Firouzi M, Bamshad MJ, Christoffels VM and Moon AM: Lethal arrhythmias in Tbx3-deficient mice reveal extreme dosage sensitivity of cardiac conduction system function and homeostasis. Proc Natl Acad Sci USA 109: E154-E163, 2012.

19. Söhl G and Willecke K: Gap junctions and the connexin protein family. Cardiovasc Res 62: 228-232, 2004.

20. Desplantez T, Dupont E, Severs NJ and Weingart R: Gap junction channels and cardiac impulse propagation. J Membr Biol 218: 13-28, 2007. 